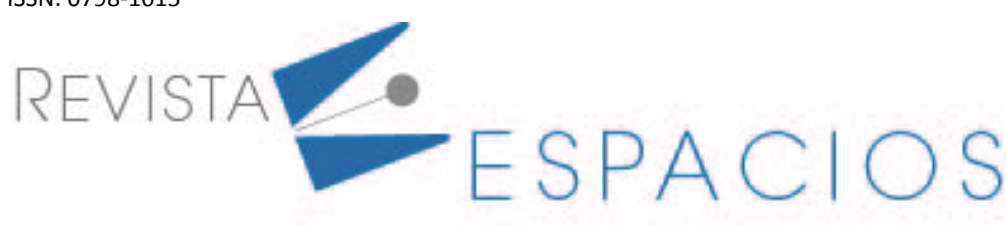

\title{
Modelo de aprendizaje de TIC en adultos
}

\section{ICT Learning Model in Adults}

\author{
FRAUSTO, Miguel A. ${ }^{1}$ \\ CORNEJO, Juan F. ${ }^{2}$
}

\begin{abstract}
Resumen
La capacitación tecnológica juega un papel muy importante en la sociedad actual, y más cuando hablamos de adultos. Por la forma de aprender y las dificultades que presentan, se llevó cabo un estudio, para conocer los factores que intervienen en el aprendizaje de las TIC en adultos. De la investigación realizada se destacan las situaciones que comprometen el aprendizaje de los adultos, para desarrollar un modelo de enseñanza que tiene como eje central la teoría del procesamiento de la información.

Palabras clave: TIC, aprendizaje, adultos, modelo.
\end{abstract}

\begin{abstract}
Technological training plays a very important role in today's society, and more so when we talk about adults, due to the way they learn and the difficulties they present, a study was carried out to know the factors that interfere in the learning of ICT in Adults. From the research carried out, the situations that compromise adult learning stand out, to develop a teaching model that has as its central axis the theory of information processing.
\end{abstract}

Key words: ICT, learning, adults, model.

\section{Introducción}

En la actualidad el aprendizaje de las nuevas Tecnologías de la Información y la Comunicación (TIC) es algo que ha tomado gran importancia, el avance tecnológico requiere que día a día nos actualicemos para poder realizar nuestras actividades cotidianas, los procesos digitales son más recurrentes en los diferentes ámbitos de nuestra vida diaria por lo que aprender a usar los diversos medios es una necesidad.

El problema no necesariamente es aprender, sino la forma de hacerlo, y y esta situación aumenta conforme a la edad, sobre todo a partir de los 40 años. De hecho, Jiménez, Lancho, Sanz y Sanz (2010) mencionan que "se ha llegado a la conclusión de que la capacidad de aprendizaje sufre un descenso con la edad" (p.119).

Fue por ello que surgió el interés de llevar a cabo el estudio sobre los problemas que presentan estos adultos en la forma de llevar a cabo el proceso de aprendizaje de las TIC, ya que su forma natural de aprendizaje tiende a ligarse con experiencias, las cuales en este caso suelen ser nulas debido a que actualmente los adultos tienen poco contacto y experiencia en el manejo de estas.

\footnotetext{
${ }^{1}$ Docente. Universidad de Guadalajara. Ing.mfrausto@gmail.com

2 Profesor Investigador Titular A. Centro Universitario del Norte. Universidad de Guadalajara. juan.cornejo@academicos.udg.mx
} 
El problema surge en el programa Comunidades de Aprendizaje y Servicios Académicos (CASA) Universitarias, que promueve la Universidad de Guadalajara $(U$ de $G)$ como punto de encuentro entre la población y la Universidad, los servicios educativos con los que cuentan estos espacios son cómputo, internet, asesoría y cursos, es a este último servicio al que está orientada la investigación, debido a que se centra más la educación tecnológica y se trabaja con el público objetivo.

La investigación se centró en los problemas de aprendizaje y las percepciones que tienen los adultos que se encuentran de entre los 18 y 55 años de edad, se convierten en el objeto de estudio con el afán de establecer algunos parámetros generales de acuerdo a sus necesidades y que esto permita brindar los medios en tecnologías para el aprendizaje además de métodos que cubra esas exigencias en la adquisición de conocimientos tecnológicos para su incorporación a la sociedad digital.

\subsection{Planteamiento del problema}

La alfabetización digital que se ha venido dando, deja experiencia en cada una de las generaciones que pasa por este proceso y parte de esta pericia consta de la observación, y entre algunos de los datos que se fueron recabando destacan que entre el $50 \%$ y $60 \%$ de los adultos que asisten a cursos de capacitación, tienen ciertas dificultades para aprender a usar un equipo de cómputo así como distintos programas de uso cotidiano (Word, PowerPoint, Excel, editores multimedia, internet, etc.), además de que 3 de cada 10 adultos, abandonan los cursos al resultarles difícil, es aquí donde se realiza la investigación con el objetivo de profundizar en el tema.

De manera general se enfatiza también la falta de lógica en el uso de herramientas informáticas, poca adaptación al trabajo en ambientes virtuales, escasa comprensión mediante explicaciones teóricas y la no visualización de los contenidos (instrucciones) para llevar a cabo una acción determinada, sin embargo, éstas son solo algunas de las dificultades que empíricamente se percibieron, la investigación del problema arrojó más datos y con mayor precisión sobre estas dificultades.

La pregunta general planteada como inicio de la investigación fue, ¿Qué dificultades presentan los adultos en el aprendizaje de las Tecnologías de la Información y la Comunicación?, las dificultades dan parte al problema y de allí surgieron los datos que dieron la pauta para tomar decisiones e implementar soluciones que en conjunto con las bases teóricas desembocaron en la propuesta del Modelo de Aprendizaje.

\subsection{Justificación}

La investigación se enfocó al sector adulto de la población de Villa Guerrero, Jalisco, México, que tiene presencia en la CASA Universitaria del municipio. Dentro del área de las tecnologías de la información y comunicación se imparte el curso de "Computación Elemental" el cual atiende a adultos de entre 18 y 55 años.

El problema de investigación tuvo como mayor justificante que el aprendizaje se pueda impartir y recibir con mayor productividad, lo cual permita que los usuarios del curso puedan transformar sus dificultades en el aprendizaje en habilidades para el desarrollo de actividades en equipos informáticos, además, los datos mencionados en el apartado anterior, representaban una cifra considerable para llevar a cabo una investigación más a fondo, tener valores estadísticos, información concreta y contundente que permitieran tener una idea más amplia de cómo atacar el problema.

Además, se cuentan con referentes en torno a que a través de las tecnologías para el aprendizaje se puede dar solución a mencionados problemas. Referente a esto Pérez (como se citó en Peralta, 2013), menciona que "la tecnología les permite hacerse de nuevas herramientas y romper el mito de que después de cierta edad ya no pueden integrarse a la tecnología". Así mismo, para ser un educador de adultos en las TIC, "hay que saber cómo 
y de qué manera aprenden las personas adultas, y dominar estrategias didácticas derivadas de la andragogía" (López, 2005, p.131-132), la ciencia del aprendizaje de adultos.

\section{Metodología}

Para llevar la investigación de la que se ha venido hablando se seleccionó el método cuantitativo, con el fin de sostener las bases hipotéticas que por observación, experiencia y frecuencia de patrones, dieron pie y son la base de esta.

A partir de los números obtenidos, se implementó un curso piloto, que apoyado en los resultados de los distintos perfiles se pueda corroborar el planteamiento del problema del que se partió, documentar las posibles circunstancias nuevas que en su momento se desconocían y finalmente presentar las consecuencias obtenidas.

La referencia anterior da pie al desarrollo de una propuesta de modelo que incluya la información recabada en la encuesta, el curso piloto y el sustento teórico, que da apoyo en el proceso de enseñanza aprendizaje de las TIC.

\subsection{Instrumento de recolección de datos}

Se diseñó en la plataforma Google Forms un cuestionario como instrumento de recolección de datos, que fue puesto a disposición de los encuestados y que se encuentra estructurado de la siguiente forma:

El cuestionario consta de 50 preguntas, que se dividen en 6 categorías:

- $\quad$ Perfil.

- $\quad$ Conocimientos de los usos de la computadora (previos y posteriores).

- Motivos de ingreso al curso.

- Evaluaciones.

- Uso de la computadora (Posteriores y actuales).

- Hacia el modelo.

Dichas categorías organizan el cuestionario de acuerdo al tipo de pregunta, las cuales permitieron definir los distintos perfiles y las situaciones adversas a las que se enfrentan los adultos en el momento de su aprendizaje, así como la posición de los participantes hacia el establecimiento de un modelo adecuado a sus necesidades.

El instrumento se organizó en una sucesión temporal, antes, durante y después, cada pregunta cuenta con opciones múltiples o en escala, según corresponde, sin embargo, una es de respuesta abierta para tomar la opinión del encuestado, esto era una opción para detectar situaciones que no habían sido consideradas y que pueden ser un factor importante a considerar, así mismo se podrían colectar sugerencias notables.

\subsection{Población y muestra}

Con el fin de conocer el avance de los estudiantes en cada curso se aplica un ejercicio práctico de evaluación al término de cada unidad (que tiene una duración aproximada de dos a cuatro semanas), mismas que se revisan y analizan para conocer cuál temática es la que presenta mayor dificultad y poderla repasar.

La muestra se obtuvo de los participantes de los cursos impartidos para adultos mismos que alcanzaron una cifra de 120 registros, dicha población con características similares en cada clase.

Para recabar la información se puso a disposición de un grupo selecto (al azar) del total de participantes, el cuestionario antes mencionado (en línea), mismo que estuvo disponible por aproximadamente cuatro meses. Dicho grupo está conformado por 35 personas que representa el $29.16 \%$ de la población total registrada. De igual manera el conjunto seleccionado se agrupó en 3 subgrupos, Grupo A (integrantes de 18 a 30 años de edad), 
Grupo B (integrantes de 31 a 42 años de edad) y Grupo C (integrantes de 43 a 55 años de edad), esto debido a la capacidad de aprendizaje de cada persona de acuerdo a su edad.

\section{Resultados y modelo}

En esta sección se mostrarán los resultados obtenidos de la aplicación del instrumento de evaluación del que se habló en el punto 2.1, así mismo se realizará un breve análisis e interpretación de cada uno de los gráficos mostrados.

Así mismo se dara a conocer el modelos desarroaldo a partir de la investigación y todos los medios teóricos que se ven involucrados en el.

\subsection{Perfil de los encuestados}

En cuanto al perfil de los usuarios estos fueron los resultados y esto amplió el panorama de quienes participaron en los distintos cursos, a continuación de presenta una gráfica con datos por edad y género.

Figura 1

\section{Edad y Género}

= a) Entre 18 y $30 \quad$ - b) Entre 31 y $42 \quad$ = c)Entre 43 y 55

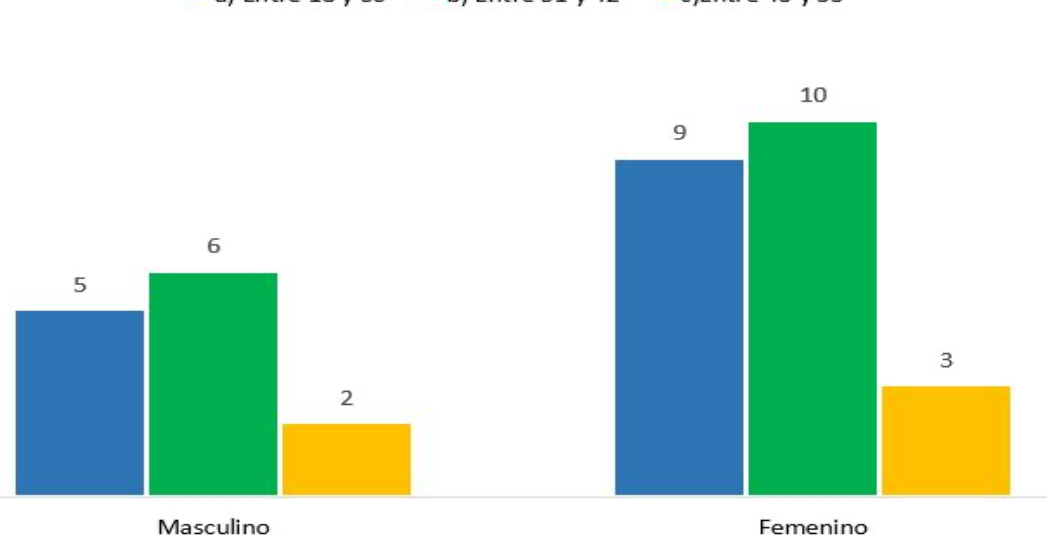

Fuente: Elaboración propia

Como se aprecia en la gráfica anterior, destaca la participación de adultos en edad intermedia entre los 31 y 42 años. De igual forma, en todos los rangos de edad sobresale la participación de las mujeres, esto último responde a que actualmente siguen siendo las madres la que apoyan a sus hijos en edad escolar y es por ello que quieren superarse personalmente. 
Figura 2

\section{Último grado de estudios por género}

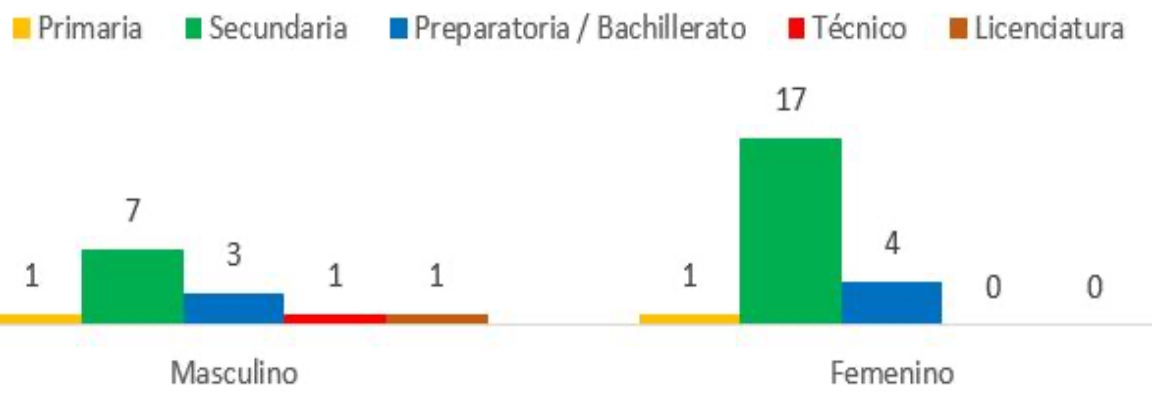

Fuente: Elaboración propia

En este caso las mayorías a destacar son nuevamente mujeres, se presentan con mayor frecuencia amas de casa, el grado máximo de estudios de nivel secundaria, de donde se resalta que estos adultos no tuvieron capacitación tecnológica en ese nivel escolar, por lo que no cuentan con bases que ayuden a ligar nuevos aprendizajes.

\subsection{Conocimientos previos y posteriores}

A continuación de analizan los conocimientos tanto antes y después del curso de capacitación, esto en algunas de las aplicaciones más populares:

Figura 3

\section{Conocimientos previos por aplicación}

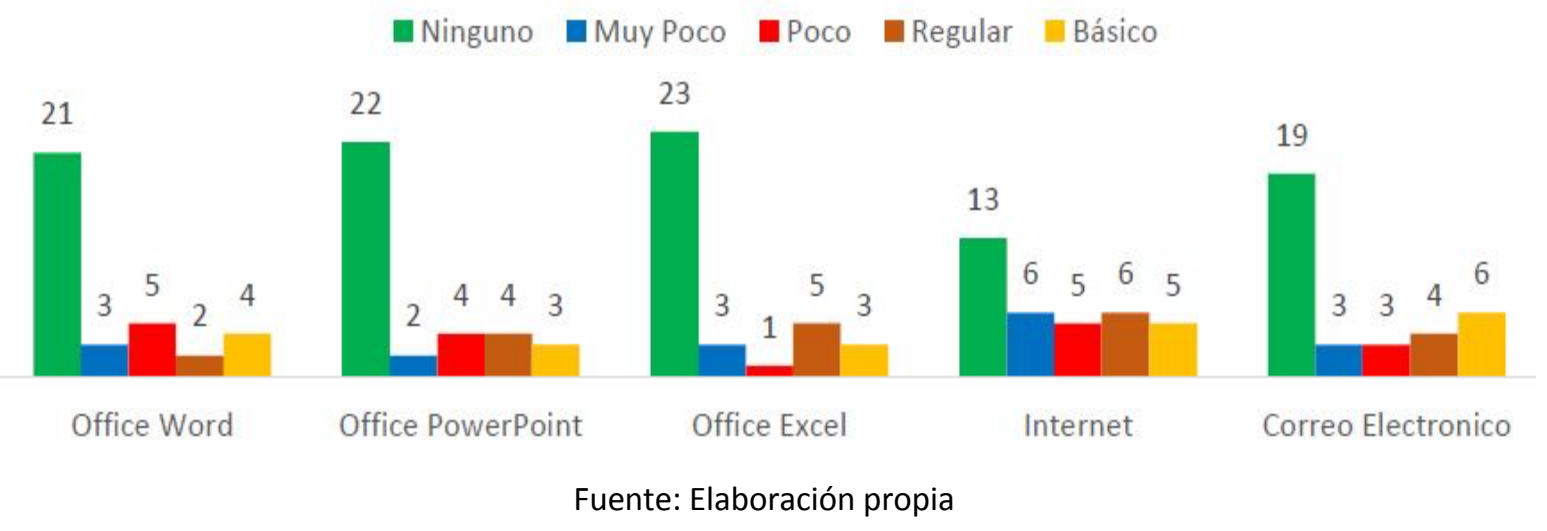


Figura 4

\section{Conocimientos posteriores por aplicación}

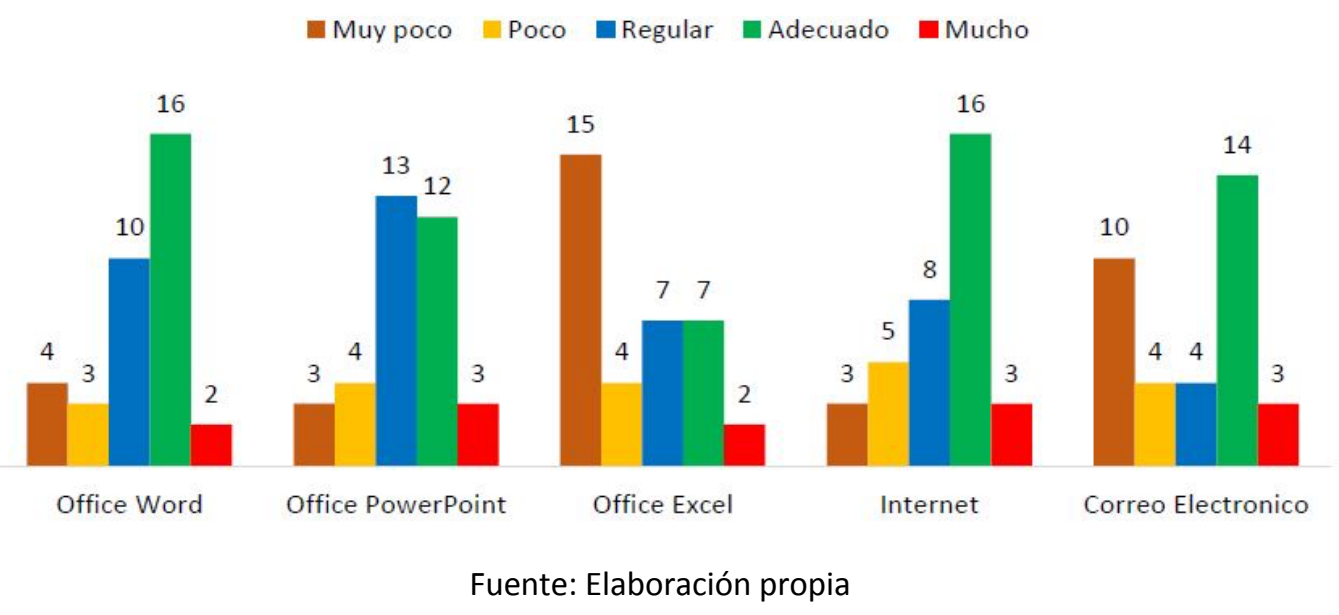

Como es de notar en las Figuras 3 y 4, la diferencia es muy evidente, en primera instancia $37 \%$ de los encuestados que mencionan no tener ningún conocimiento previo de manera general, ante $40 \%$ y $26 \%$ que responden a egresar del curso con conocimientos adecuados o mucho respectivamente, la suma de estos valores (66 \%) es de considerar ya que se rebasa la media del $50 \%$.

Solo $34 \%$ egresan con cocimientos de regular a muy poco, es decir $3 \%$ menos de aquellos que ingresaron sin tener saberes previos. De igual manera es de resaltar la mejora que existe por aplicación, los números de "ninguno" y "muy poco" en las gráficas de conocimientos previos y posteriores bajan de una manera considerable lo que significa que de tener un promedio de 19.6 de personas que no contaban con conocimientos previos, después del curso se cuenta con un cociente de al menos 7 con un aprendizaje mínimo.

Incluso el hecho de aprender a usar algunas tecnologías abre la puerta a la adquisición de estas, como se muestra a continuación.

Figura 5

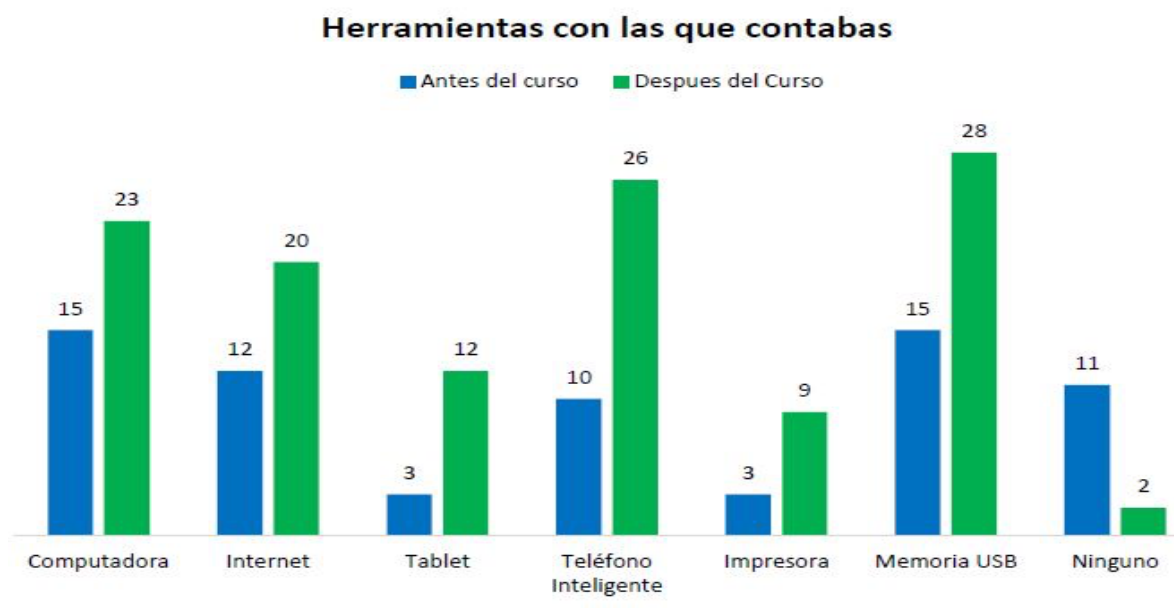

Fuente: Elaboración propia

Como se puede observar los números después del curso superan al antes, este indicio es relevante, se muestra que al aprender a usar las tecnologías, los adultos tienden a invertir con menos temor, ya que sirve no solo para los hijos si no que ahora ellos podrán controlar y saber que actividades realizan e incluso apoyarlos en lo que requieran. 


\subsection{Dificultades de aprendizaje}

Ahora se muestran los resultados de una de las partes más importantes y motivo de la investigación realizada, las dificultades que se presentan en el aprendizaje de TIC en Adultos.

Figura 6

\section{aprendizaje \\ = Falta de experiencia $=$ Dificultad \\ - Familia \\ - Presión \\ - Trabajo \\ - Ninguno}

Factores que interferían en tu

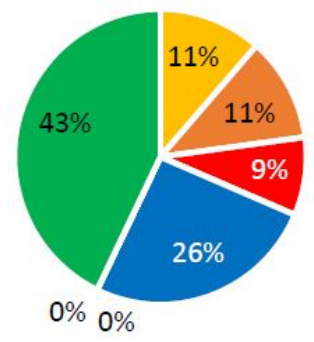

Fuente: Elaboración propia
Figura 7

\section{El entorno de trabajo (lo que vez en pantalla) ¿'Te resultaba un obstáculo en tu aprendizaje?}

" Sí = No - Algunas veces

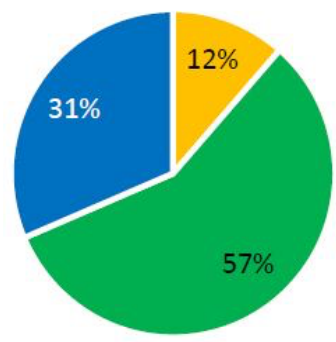

Fuente: Elaboración propia.

En la investigación se detectó que al menos $64 \%$ de quienes toman un curso sufren de un factor que interfiere en su aprendizaje. En la segunda grafica hay un valor positivo ( $57 \%$ no tienen problemas con el entorno de trabajo en la pantalla de una computadora), aunque el $43 \%$ si los tienen. Se analizó este último por separado debido a que es algo recurrente y que se da por observación y los resultados indican que hay un problema a resolver.

En la figura 8 se muestra una gráfica respecto a la edad como factor que interfiere en el aprendizaje, como sabemos ya, la capacidad de aprendizaje se reduce conforme la edad avanza, pero como se observa, los encuestados no consideran que esta sea un factor en contra de su aprendizaje, más es de mencionar que si es de ponerle atención. 
Figura 8

Conocimientos posteriores por aplicación

¿Crees que tu edad es un obstáculo para tu aprendizaje?

- Si - Probablemente "No = Nolo sé

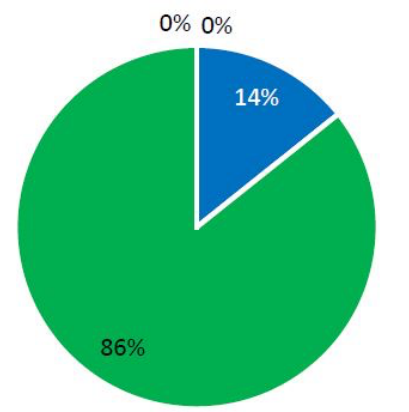

Fuente: Elaboración propia

\subsection{Hacia el modelo}

Para conocer la opinión de los encuestados sobre el desarrollo de un modelo de aprendizaje se realizaron varias preguntas que arrojaron resultados positivos para llevar a cabo el modelo de aprendizaje, dicha derivación arrojo los siguientes números.

Tabla 1

Hacia el modelo

\begin{tabular}{|c|c|c|c|c|}
\hline \multirow[b]{2}{*}{ Preguntas } & \multicolumn{4}{|c|}{ Respuestas y porcentajes } \\
\hline & Si & No & Probablemente & No lo sé \\
\hline $\begin{array}{l}\text { ¿Crees que si participas en un curso hecho a tu } \\
\text { gusto tendrías mejor desempeño? }\end{array}$ & $71 \%$ & $\mathrm{n} / \mathrm{a}$ & $26 \%$ & $3 \%$ \\
\hline $\begin{array}{l}\text { ¿Crees que tu opinión antes de iniciar el curso } \\
\text { sería importante para evitar los factores que } \\
\text { interfieren con tu aprendizaje? }\end{array}$ & $63 \%$ & $17 \%$ & $11 \%$ & $9 \%$ \\
\hline $\begin{array}{l}\text { ¿Te gustaría participar en un curso creado a tu } \\
\text { gusto? }\end{array}$ & $77 \%$ & $3 \%$ & $20 \%$ & $\mathrm{n} / \mathrm{a}$ \\
\hline $\begin{array}{l}\text { ¿Crees que si participas en un curso hecho a tu } \\
\text { gusto tendrías mejor desempeño? }\end{array}$ & $71 \%$ & $\mathrm{n} / \mathrm{a}$ & $26 \%$ & $3 \%$ \\
\hline $\begin{array}{l}\text { ¿Crees que es necesario crear programas de } \\
\text { estudio de acuerdo a las necesidades de los } \\
\text { participantes? }\end{array}$ & $100 \%$ & $0 \%$ & $\mathrm{n} / \mathrm{a}$ & $\mathrm{n} / \mathrm{a}$ \\
\hline
\end{tabular}

* n/a = No aplica respuesta a la pregunta

Fuente: Elaboración propia

La participación de los encuestados demostró que un cambio era necesario, el desarrollo del Modelo de aprendizaje de TIC en Adultos tenía que seguir el cauce, por lo que contando con las herramientas necesarias se procedió a desarrollar dicho modelo el cual se explica a detalle en la siguiente sección.

\subsection{Desarrollo del modelo de aprendizaje}

Para Girona, Guárdia, Mas \& Sangrá, "Los diferentes modelos de aprendizaje son el resultado una metodología orientada para que el estudiante aprenda de un modo determinado" (2005, p. 42), si bien se cuentan con 5 
modelos de aprendizaje generales, se ha estado trabajando en adaptar o desarrollar modelos de acuerdo a las necesidades de los alumnos, la edad, lo que estudian y lo más importante la forma de aprender

De lo anterior que Girona et al mencionan que "La selección del modelo de aprendizaje del material tiene repercusiones más allá de la misma estructura y condiciona la metodología global de toda la acción formativa" (2005, p. 42 - 43). Como describen los autores las consecuencias del paradigma recaen en la acción formativa, es por ellos que universidades de todo el mundo cada vez se enfocan en el desarrollo de formas, estrategias y métodos que ayuden a sus estudiantes a ser más productivos tanto académica como laboralmente.

A continuación, se detallan las bases teóricas usadas y que en conjunto con los resultados del estudio realizado dieron como resultado el Modelo de aprendizaje de TIC para adultos propuesto en este artículo.

\subsection{Estrutura del modelo}

La propuesta está basada y estructurada con tres elementos, primero la experiencia en la capacitación tecnológica con adultos seguido de las Leyes y Principios del Aprendizaje y finalmente la Teoría del Procesamiento de la Información de la que se destaca cada una de las etapas del modelo, mismas que se representan y explican a continuación.

\subsubsection{Pre - aprendizaje}

En esta etapa se recaba la información de adultos con los que se estará trabajando, esta fase es para el asesor y de ella depende el buen desenvolvimiento y seguimiento del curso.

Esta fase se conforma de tres partes, la primera: intereses de los adultos, los cuales se conocerán a través de encuestas, el resultado de éstas permitirá la creación de grupos afines, una demanda por parte de los adultos.

En cuanto al Uso de las TIC (segunda parte) donde se implementan las tecnologías que se van a usar, ahora no solo en cuestiones de una computadora sino también de tecnologías móviles, se definen estrategias de aprendizaje y temáticas de acuerdo a las necesidades y Propuestas de los participantes (tercera parte).

Figura 9

Etapa de Pre - Aprendizaje

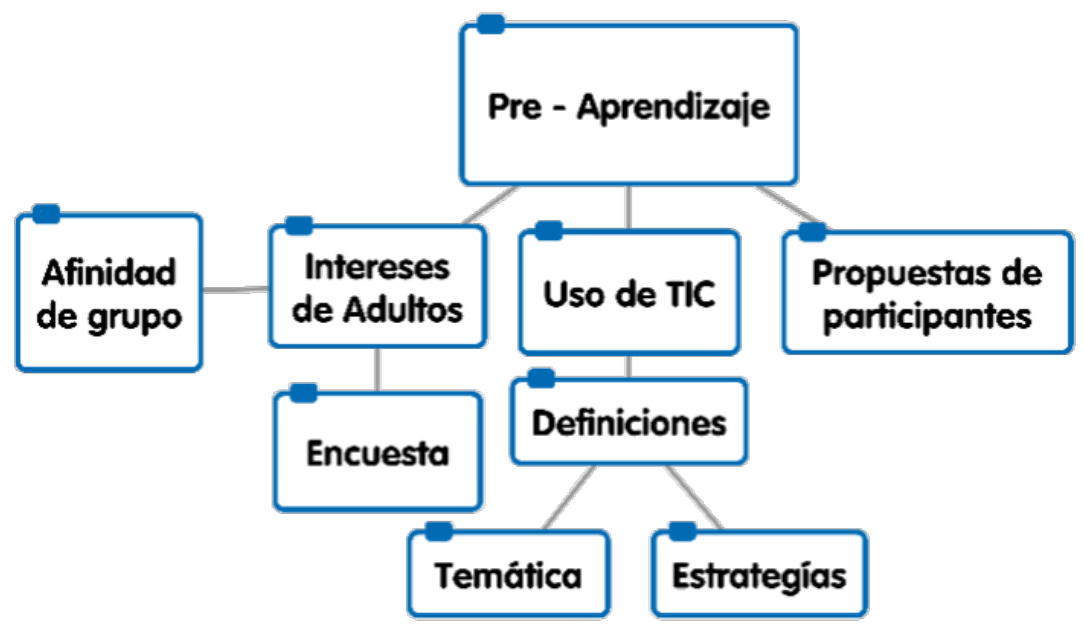

Fuente: Elaboración propia

Toda la información necesaria en esta etapa se recoge previo al inicio del curso, puede ser incluso cuando se asiste a inscribirse o registrarse, todas las opciones se van juntando y con ello se define el programa de estudio. 


\subsubsection{Codificación}

Esta etapa al igual que las demás tomadas de la teoría del procesamiento de la información, la cual según Shuell (1986) "se enfocan en la manera en que las personas ponen atención a los eventos que ocurren en el ambiente, codifican la información que deben aprender, la relacionan con los conocimientos que tienen en la memoria, almacenan el conocimiento nuevo en la memoria y lo recuperan a medida que lo necesitan" (como se citó en Schunk, 2012, p. 164)."

Para el caso de la emisión de la información en esta etapa se toma en cuenta las leyes y principios de aprendizaje en adultos, los cuales son básicos e importantes para un programa de educación continua según Escobar (1978) para llevar a cabo una estrategia menos rígida y acorde a la zona donde son aplicados. Con esto se definen los recursos y cómo se explicarán los conocimientos, mostrando mediante proyecciones, tal y como lo verá el alumno en su práctica, teniendo en cuenta los recursos multimedia que pueden ser de apoyo para brindar explicaciones, es importante utilizar la demostración - práctica, "primero se ve y aprende como se hace, luego se hace, posteriormente se enseña a hacerlo (siguiente etapa)".

Para que la recepción sea fructífera es necesario una información organizada y con una secuencia, para que el alumno pueda elaborarla, es decir, llevarla a cabo para aprenderla y apropiarse de ella, para esto deberá usar distintos métodos para esquematizar los datos, pueden ser dibujos, notas e incluso fotografías (el método que seleccione cada educando será el mejor) esto le servirá para recuperar información que llegue a olvidar.

Figura 10

Etapa de codificación

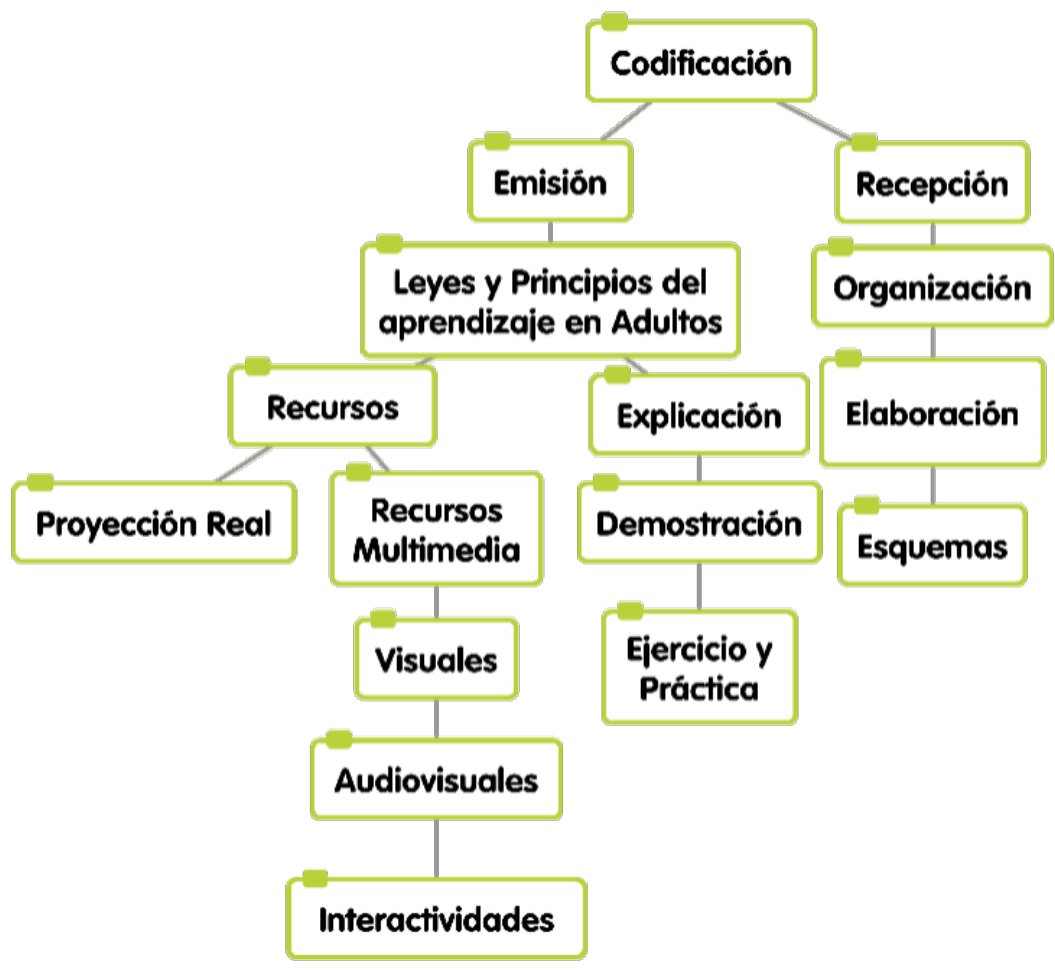

Fuente: Elaboración propia

\subsubsection{Almacenamiento}

Esta etapa es importante y en cierto grado complicada de lograr, ya que consiste en que el adulto almacene en Memoria a Largo Plazo (MLP) la información que ya ha recibido para que no la olvide. 
Cuando una persona aprende TIC sin haber tenido antes contacto, no hay información base para vincular los nuevos aprendizajes, pero si espacio para almacenarlos, por lo tanto, la mejor manera de iniciar a crear esos vínculos es mediante la explicación y repaso, la nueva información que entrará en la memoria de la persona fungirá como anclajes para la nueva información que siga agregando.

Con los adultos es permanecer propiciando el aprendizaje, para lograrlo, las prácticas objetivas y significativas deben ser un quehacer diario, por supuesto la motivación juega un papel muy importante, y por solicitud de los adultos, disponer de espacios para los que ya han aprendido puedan compartirlo con los rezagados, una práctica muy útil ya que en ocasiones la codificación entre alumnos se da mejor que incluso de la forma asesor - alumno.

Figura 11

Etapa de Almacenamiento

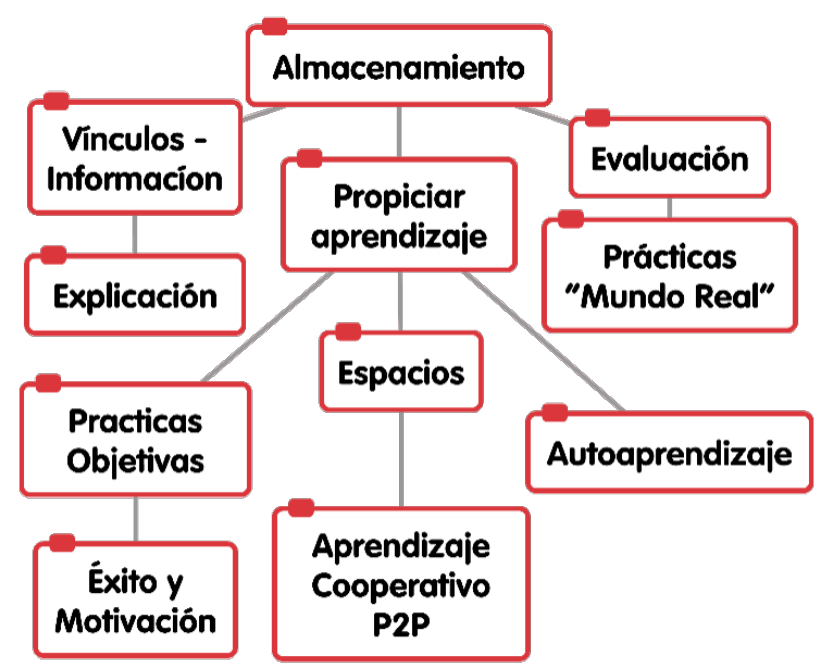

Fuente: Elaboración propia

El autoaprendizaje es algo a inculcar. El que los adultos formen sus conocimientos mediante la exploración es vital para que vayan desarrollando nuevas habilidades, que deberán ser evaluadas con prácticas aplicadas a la vida diaria.

\subsubsection{Recuperación y olvido}

Figura 12

Etapa de Recuperación y Olvido

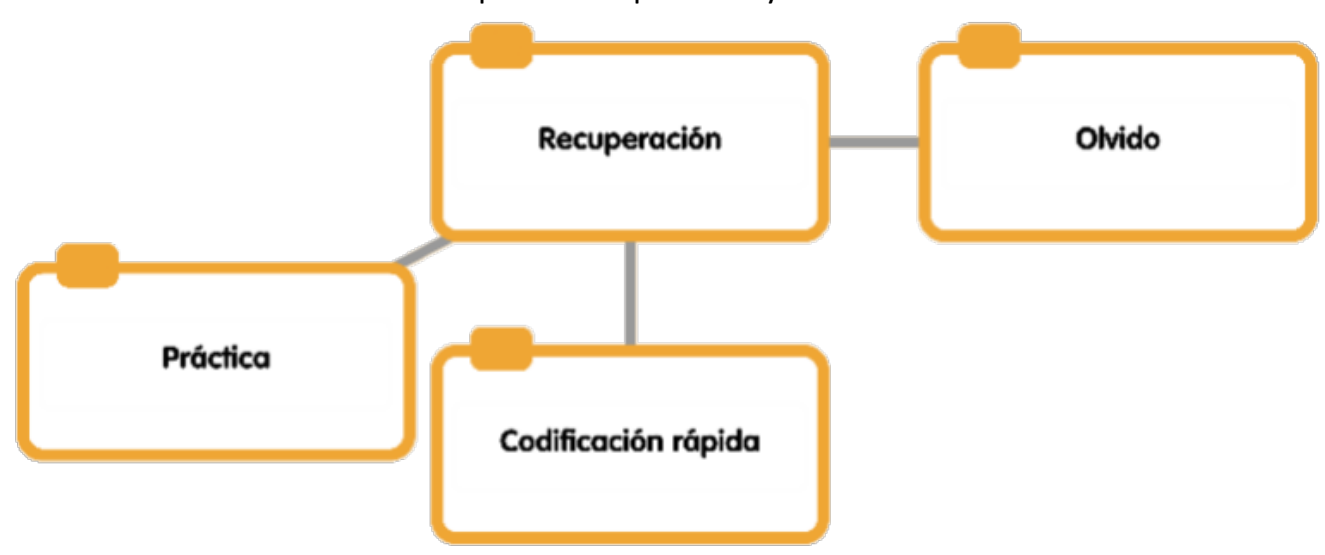

Fuente: Elaboración propia 
Esta etapa está vinculada mediante la práctica con los conocimientos ya adquiridos. Es decir, seguir practicando para no olvidar y lo que se practicó seguirlo aplicando para no olvidar, por eso hay una auto-relación en la parte de práctica (Figura 12), en caso de que se presente el olvido será necesario recordar mediante una codificación rápida o repaso para recuperar la información.

Todas las etapas están relacionadas entre sí y en algunas partes que las componen, por tal motivo se muestra a continuación el modelo completo.

\subsubsection{Modelo de aprendizaje de TIC en adultos}

Figura 13

Modelo de aprendizaje de TIC en Adultos

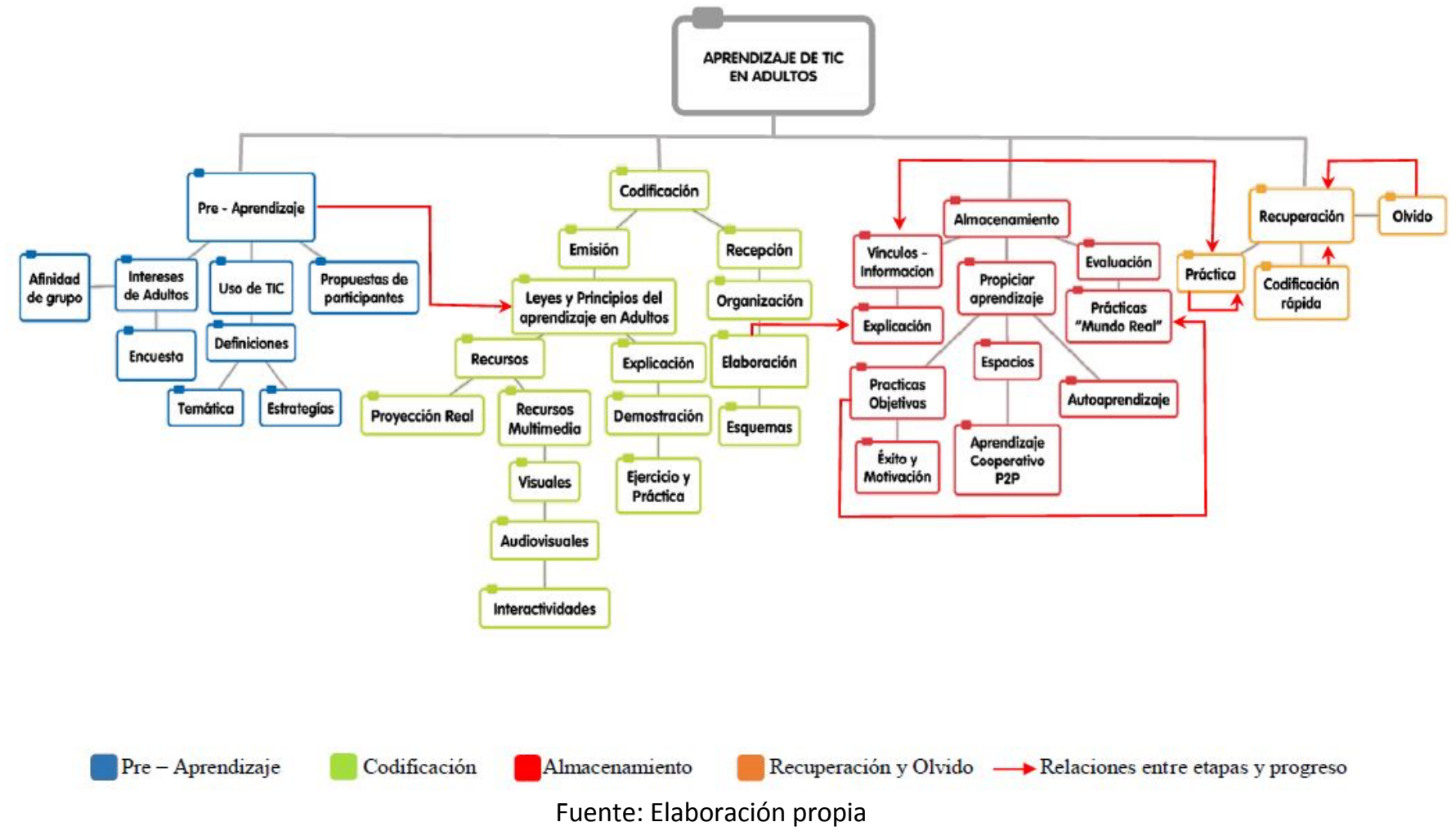

3.6.6. Bases teóricas del modelo

\section{a) Teoría del Procesamiento de la Información}

La Teoría del procesamiento de la información es una de las teorías del aprendizaje que sirve de apoyo para identificar los aspectos específicos que apoyen a saber cuál es la forma en que aprenden los adultos, sector social en el cual está enfocado el problema de investigación y también qué dificultades presentan en su aprendizaje, para tener dicha información como base y partir de ella.

En el modelo se establecieron etapas que son rescatadas de la teoría del procesamiento de la información, que explican a grandes rasgos el aprendizaje y manejo de información en las personas. La primera de estas etapas es la codificación, es decir la interpretación de representaciones, concretamente Schunk (2012) define codificación como:

El proceso de colocar la nueva información en el sistema de procesamiento y prepararla para almacenarla en la MLP (.Memoria a Largo Plazo) Por lo general la codificación se consigue dándole un significado a la nueva información e integrándola a la información conocida en la MLP. (p. 187) 
La siguiente etapa que surge de esta teoría es el almacenamiento la cual según Schunk (2012), se describe de la siguiente manera:

Primero, el aprendiz recibe información nueva, como ocurre cuando el profesor hace una afirmación o el estudiante lee una oración. Luego, la información nueva se traduce en una o más proposiciones en la MT (Memoria de Trabajo o Memoria a Corto Plazo) del sujeto. Al mismo tiempo, se indican las proposiciones relacionadas de la MLP. Las nuevas proposiciones se asocian con las proposiciones relacionadas en la MT mediante el proceso por el cual se difunde la activación (p. 193).

Entendemos también por proposiciones como oraciones o afirmaciones, en simples palabras la secuencia que describe Schunk, la podemos relacionar en el proceso de enseñanza aprendizaje simple, el profesor le brinda información al alumno (o el aprendiz extrae dicha información de cualquier fuente de consulta), el formado traduce o codifica mediante el proceso visto anteriormente toda esa información, en ese mismo instante el educando esta relacionando lo visto con la información con la que ya contaba y la relaciona directamente con el ámbito al que pertenece, sabiendo de inmediato hacia qué área del conocimiento está orientada esa información, la información nueva y con la que ya contaba (proposiciones) se relacionan y almacena en la memoria del individuo.

Aunque suena como un proceso simple, en realidad es un proceso muy difícil de lograr y más aún cuando existen distractores o dificultades en el aprendizaje. Es la etapa más importante ya que de lograr que la información se almacene en nuestra mente podemos decir que el aprendizaje ha sido exitoso. Sin embargo, si la práctica de dichos aprendizajes se deja de dar, la información almacenada tiende a olvidarse y es cuando viene la siguiente etapa. En caso de la que la información se mantenga en la MT (Memoria de Trabajo), y no se almacene de manera adecuada en la MLP, el alumno se enfrenta a un olvido casi inmediato, hablando de los adultos este olvido se puede llevar incluso en un lapso no mayor a una hora, quizá vio como se hace un procedimiento lo practicó, pero después de un momento su mente lo deseche y cuando requiera nuevamente hacer el procedimiento simplemente ya no recuerde como hacerlo.

La última etapa del proceso de aprendizaje es la recuperación y el olvido. ¿Cómo se recupera la información cuando se le solicita a un estudiante?, por ejemplo cuando se le hace una pregunta, Anderson (como se citó en Schunk, 2012) menciona el siguiente proceso:

La pregunta ingresa a su MT y se separa en proposiciones. El proceso mediante el cual ocurre esto tiene una base neurológica aún no bien comprendida, pero las evidencias indican que, mediante la difusión de la activación, el material activa la información asociada en las redes de memoria para determinar si responde la pregunta. Si la contesta, esa información se traduce en un enunciado y se verbaliza al interrogador o se convierte en patrones motores para escribirla. Si las proposiciones activadas no responden la pregunta, la activación se difunde hasta encontrar la respuesta. Cuando no hay tiempo suficiente para que la difusión de la activación localice la respuesta, los estudiantes pueden hacer una conjetura educada. (Ídem, p. 200)

Es un proceso mental complejo pero que a la vez se realiza de una manera muy rápida, el autor comenta sobre la difusión de activación, en si se trata del proceso de cómo vincular la información entrante con la que ya se almacenó en memoria a largo plazo, con este proceso el estudiante relaciona la pregunta con la respuesta que piensa dar, ya sea contestando verbalmente, escribiendo o incluso dibujado, si no contesta correctamente el proceso se inicia de nuevo para buscar otra respuesta o si no la tiene, el estudiante puede crear (imaginar) un juicio que pueda responder a la pregunta.

Este proceso resulta interesante, en múltiples ocasiones las personas saben cómo hacer las cosas, más no las recuerdan, necesitan que se les indique cómo empezar, en este problema de investigación por la experiencia adquirida (propia), los adultos tienden a responder un "no sé", sin embargo, al comenzar a explicar, pronto la 
difusión de activación hace que encuentre la respuesta y puedan realizar o contestar lo que se les ha solicitado, esto claro si el olvido no ha sido a largo plazo, si es en corto, como se mencionó anteriormente, es posible que la persona recupere la información y logre realizar su cometido.

La recuperación de información, depende de las demás etapas del aprendizaje y no necesariamente debe considerarse olvido.

\section{b) Leyes y Principios del aprendizaje en adultos}

Las leyes del aprendizaje son tres claves de apoyo para desarrollar en el educando una instrucción funcional.

Moreno (1988, p.1) enuncia las siguientes leyes:

- Ley de la preparación: La necesidad y el deseo de aprender pone en funcionamiento las habilidades necesarias.

- La ley del efecto: a más éxito, se obtiene más motivación y más placer del aprendizaje.

- La ley del ejercicio: la práctica hace al maestro, a más actividad, más aprendizaje.

Esto tres enunciados mencionan la importancia de prepararse, pero esto se debe de dar de acuerdo a un deseo que viene de una necesidad por obtener nuevas habilidades que permitan un desenvolvimiento mejor en un área dentro de las actividades cotidianas.

La segunda ley menciona la importancia de la motivación para seguir consiguiendo el deseo de aprender por parte del educando y que este siga adelante en su capacitación y no la abandone.

La tercera ley hace mención de un dicho popular, donde la práctica será directamente proporcional al aprendizaje funcional que adquiera el educando.

\section{c) Leyes y Principios del aprendizaje en adultos}

Estos principios son pasos y pistas para la capacitación en adultos, son un guía que permite lograr un mejor desempeño en el proceso de enseñanza aprendizaje con las personas.

Moreno (1988, p.1) enuncia los siguientes principios:

- Los adultos son autónomos y auto-dirigidos.

- Los adultos traen creencias establecidas, valores y opiniones a cualquier entorno de aprendizaje.

- La mayoría de los alumnos adultos están orientados a los objetivos.

- Los alumnos adultos prefieren temas relevantes.

- Los adultos aprenden de sus errores, pero son extremadamente sensibles al fracaso.

- Los adultos gustan de aprender información práctica.

- Los adultos prefieren aprender a través de los diferentes sentidos.

- Los adultos aprenden mejor cuando tienen éxito. Hay que asegurarse de proveer un "aprendizaje exitoso".

- Los adultos aprenden mejor cuando pueden monitorear su propio progreso.

- Los adultos aprenden mejor cuando los contenidos del aprendizaje se han programado para adaptarse a ellos.

- Los adultos tienden a centrarse en problemas cuando enfrentan el aprendizaje.

Como es de notar, cada enunciado menciona un aspecto importante a considerar cuando se capacite a los adultos y ayudan al asesor a tener los fundamentos para guiar por buen camino el aprendizaje de los formados. 


\section{Conclusiones}

El presente trabajo reporta una investigación cuyo objetivo primordial fue el iniciar a crear un modelo de aprendizaje de TIC en adultos, pensando en sus necesidades y dificultades de aprendizaje, así como los factores externos que intervienen de forma negativa en este proceso.

Si se habla del perfil de los encuestados se destaca notablemente la participación mayoritaria por parte de las mujeres, aunque en su mayoría entran a un curso de capacitación tecnológica por superarse de manera personal, otro de los factores que también influye de acuerdo a la experiencia, son los hijos, el poder apoyarlos en sus actividades académicas.

El nivel máximo de estudio en la mayoría de los encuestados es de secundaria, al considerar de igual manera que esa parte tienen edades entre los 31 y 42 años, lo que permite deducir que no tuvieron capacitación tecnológica, el hecho de estar familiarizándose con las nuevas tecnologías involucra un esfuerzo mayor ya que no cuentan con antecedentes para vincular los nuevos aprendizajes.

En cuanto a capacitación tecnológica se muestra realmente que hay un avance significativo, es claro, se puede mejorar y hay que perfeccionar, las cifras indican en primer lugar que con el curso se reduce el número de personas que ingresan sin conocimientos previos, pero, ¿Qué significa realmente esto?, la respuesta es que una persona que no sabía ni encender un equipo de cómputo, al menos egresa sabiendo cómo encender y apagar además de conocer el funcionamiento básico de la computadora como herramienta, ya se ha forjado un base, será necesario que estas personas se sigan capacitando, para que sigan elaborando sus conocimientos con antecedentes, el siguiente paso en su capacitación será más sencillo.

La contraparte es el $66 \%$ que adquieren conocimientos adecuados o mencionan aprendieron suficiente, si este porcentaje de personas lo consideran así, otros pueden llegar a alcanzar este mismo nivel, es por ello que se ha elaborado una propuesta que permite en las diferentes etapas y a través de los diferentes medios y aportaciones que han realizado los participantes que ese aprendizaje sea más eficiente.

Una de las formas de lograr lo antes mencionado es que si un adulto aprende a utilizar una aplicación en la computadora que le ayude a desarrollarse en las actividades cotidianas que así lo requieran, tendrá un mejor desempeño, centrara su atención porque le interesa aprender por lo tanto tendrá una mejor apropiación del conocimiento. Pero si el aprendizaje que está adquiriendo no representa servicio a su favor, y no sabe cómo lo implementará en las actividades que desempeña, le resultará de mayor dificultad aprender dicha aplicación.

En el proceso de aprendizaje los adultos se enfrentan a diferentes dificultades, se hacer notar que $64 \%$ de los encuestados tuvieron un conflictos, tales como: trabajo, familia, dificultad de uso y experiencia, algo que es de esperarse al trabajar con adultos, incluso algunos padecen más de una dificultad, es por ello que en la propuesta de modelo se dedicó espacio a la opinión previa al curso, con lo cual se pueden crear horarios adecuados para evitar que el trabajo se convierta en un factor que impida asistir y así retrasarse en su aprendizaje, además se puede obtener la propuesta de acuerdo a los días que pueden asistir y que tienen compromisos menores con la familia.

Para el caso de la experiencia y dificultad se establecieron espacios para repasar con ayuda del asesor y/o un compañero, lo que permite abonar la experiencia de los más avanzados con lo que aprenden más lentamente y que pueden llegar a entenderse incluso mejor que con las palabras del asesor.

Finalmente, se confirmó el problema que tienen algunas personas (42 \%) para entender el entorno de trabajo en la pantalla de una computadora, este problema surgió de la observación y se confirma con el estudio realizado. 
Las dificultades presentadas llegan a concatenar toda la información que brindaron los encuestados, la experiencia docente, la observación y las bases teóricas para desarrollar un modelo de aprendizaje de TIC para adultos, que de acuerdo al estudio, es necesario que existan temáticas que sean de su interés y con este análisis, se confirmó esta exigencia que ya se había sustentado teóricamente con el aprendizaje significativo, lo que supuso un gran avance en la investigación cuyos resultados complementan esta idea.

Se comprobó que los adultos creen que al estudiar cosas que sean de su pleno interés y que tengan que ver con sus actividades cotidianas mejorará el desempeño que estos tengan, además podrán incorporarse de mejor manera a la sociedad digital en la que vivimos.

La opinión de los participantes dio paso a crear un modelo, una tendencia que ya se ha venido dando en diferentes universidades nacionales e internacionales, prestando mayor atención a un aprendizaje académico humanista.

Se determina que los tipos de aprendizaje de acuerdo a las bases teóricas influyen bastante en la forma de aprender, dentro de las respuestas abiertas aplicadas a los encuestados, se sugirió la formación de grupos afines que aprenden de manera similar, muchas veces es algo complicado por la cantidad de registros o cupos.

Se describen que los problemas detectados son realmente una complicación a resolver, y que cada uno debe ser atendido de forma apropiada, con método y explicación basada en la vida cotidiana. Que la edad no es una limitante si no que tiene que ser tratada de distinta forma a cada grupo de edad y es una realidad confirmada en su totalidad por quienes participaron en la encuesta.

La inclusión digital está en la forma de impartirla, ofreciendo herramientas tecnológicas, si, las mismas, no hay que buscar más, internet, navegadores web (Google Chrome, Mozilla FireFox, Edge), ofimática (Microsoft Office o Libre Office por ser las más populares), multimedia (editores sencillos, intuitivos y de fácil uso), usando las herramientas más simples y necesarias, sin incluir aquellas que no son de relevancia o significado para los educandos. Es necesario mejorar los recursos, interactividades, audiovisuales, proyecciones acompañadas con una buena explicación, las veces que sean necesarias, la edad de cada grupo nos dirá cuentas veces hay que repetir, generar prácticas amables acordes a los motivos de cada participante y recordar, siempre estar repasando lo aprendido, esa es la forma para lograr un aprendizaje de TIC eficiente y una inclusión digital exitosa.

\section{Referencias bibliográficas}

Escobar, F. (1978). Principios del aprendizaje del adulto. El uso de las teorías de aprendizaje en el adulto. Recuperado el 25 de Abril de 2019, de http://hist.library.paho.org/Spanish/EMS/4946.pdf

Frausto, M. A. (2018). El aprendizaje de las TIC en adultos: una perspectiva desde CASA Universitaria Villa Guerrero. (Tesis de maestría). Universidad de Guadalajara, Jalisco, México.

Girona, C., Guárdia, L., Mas, X., \& Albert, S. (2005). Los modelos de aprendizaje. En C. Girona, L. Guárdia, \& S. A. Mas Xavier, Los materiales de aprendizaje en contextos educativos virtuales (págs. 42-43). Barcelona: Editorial UOC.

Jiménez Frías, R., Lancho Prudencio, J., Sanz Bachiller, J. C., \& Sanz Fernández, F. (2010). Fundamentos psicológicos del aprendizaje adulto. En R. J. Frías, J. L. Prudencio, J. C. Bachiller, \& F. S. Fernández, Educación de personas adultos en el marco del aprendizaje a lo largo de la vida (pág. 119). Madrid, España: Editorial UNED.

López Camps, J. (2005). Aprendizaje de Adultos. En J. López Camps, Planificar la formación con calidad (págs. 131-132). WK Educación. Obtenido de 
https://books.google.com.mx/books?id=DYn6IgLhoToC\&lpg=PA131\&ots=hiTPJZF5to\&dq=Los\%20ni\%C3\%B 1os\%20aprenden\%20explorando\%20e\%20interrelacionando\%20con\%20su\%20entorno.\%20Los\%20j\%C3\% B3venes\%2C\%20a\%20diferencia\%20de\%20los\%20ni\%C3\%B1os\%2C\%20ya\%20piensan\%20de\%20

Moreno, P. M. (1998). Biblioteca Daniel Cosío Villegas. Recuperado el 05 de Noviembre de 2018, de http://biblio.colmex.mx/curso_formacion_formadores/adultlearn.pdf

Peralta, L. (1 de Octubre de 2013). CNN México. Recuperado el 17 de Marzo de 2015, de http://mexico.cnn.com/salud/2013/10/01/los-adultos-mayores-del-analfabetismo-tecnologico-a-laadopcion-digital

Schunk, D. H. (2012). Teoría del Procesamiento de la Infromación. En D. H. Schunk, \& M. Vega Pérez (Ed.), TEORÍAS DEL APRENDIZAJE (L. E. Pineda Ayala, \& M. E. Ortiz Salinas, Trads., SEXTA ed., págs. 10-217). Naucalpan de Juárez, Estado de México, México: PEARSON EDUCACIÓN.

Esta obra está bajo una Licencia Creative Commons Attribución-NoCommercial 4.0 International

(cc) BY-NC 